\title{
Una práctica a analizar: La consulta previa de proyectos de inversión en el sector eléctrico
}

\author{
Milagros Mutsios Ramsay*
}

\begin{abstract}
Resumen. - Este año se cumplen 25 años desde que el Perú ratificó el Convenio No. 169 de la Organización Internacional del Trabajo (OIT), sobre los Derechos de los Pueblos Indígenas y Tribales. Este Convenio tuvo por objetivo, entre otros, el reconocer derechos fundamentales a los pueblos indígenas vinculados con su participación en la vida política de un determinado país. Considerando lo anterior, la polémica sobre la aplicación del procedimiento de consulta previa para proyectos de inversión ha tomado una especial relevancia respecto a su análisis y desarrollo tanto a nivel doctrinal como jurisprudencial. En este contexto, el presente artículo buscará brevemente desarrollar las características del derecho a la consulta y su aplicación en la actualidad. Ello con el objetivo de extrapolar esta aplicación al sector eléctrico, diferenciando los casos en los que se ha ejecutado esta consulta y cuáles no, permitiendo así cuanto menos determinar los aspectos a mejorar y profundizar para la aplicación efectiva y eficiente de este derecho.
\end{abstract}

\begin{abstract}
This year marks 25 years since Peru ratified International Labour Organization (ILO) Convention No. 169 on the Rights of Indigenous and Tribal Peoples. The objective of this Convention was, inter alia, to recognize the fundamental rights of indigenous peoples linked to their participation in the political life of a given country. In view of the foregoing, the controversy over the application of the prior consultation procedure for investment projects has taken on special relevance with regard to its analysis and development at both the doctrinal and jurisprudential levels. In this context, this article will briefly seek to develop the characteristics of the right to consultation and its current application. The objective is to extrapolate this application to the electricity sector, differentiating the cases in which this consultation has been carried out and which have not, thus allowing us to determine at least the aspects to be improved and deepened for the effective and efficient application of this right.
\end{abstract}

Palabras claves. - Consulta previa - Proyectos eléctricos - Convenio $N^{0} 169$ de la OIT.

Keywords. - Prior consultation - Electrical projects - ILO Convention No 169. 


\section{Alcance del derecho a la consulta previa y su regulación aplicable al sector eléctrico}

Como lo hemos indicado en líneas arriba, el derecho a la consulta previa fue incorporado en la legislación peruana a través del Convenio No. 169 de la OIT (el, “Convenio 169"). Así, el Convenio 169 fue aprobado por la Resolución Legislativa No. 26253 del 5 de diciembre de 1993, en cumplimiento de lo dispuesto en el artículo 56 de la Constitución Política del Perú1. Posteriormente, fue ratificado el 17 de enero de 1994, entrando en vigencia el 2 de enero de 1995 y, a partir de esa fecha, forma parte de la legislación en aplicación del artículo 55 de la Constitución².

El artículo 6 del Convenio 169 regula que los gobiernos deberán “(a) consultar a los pueblos interesados, mediante procedimientos apropiados y en particular a través de sus instituciones representativas, cada vez que se prevean medidas legislativas o administrativas susceptibles de afectarles directamente [...] Las consultas llevadas a cabo en aplicación de este Convenio deberán efectuarse de buena fe y de una manera apropiada a las circunstancias, con la finalidad de llegar a un acuerdo o lograr el consentimiento acerca de las medidas propuestas". (énfasis agregado)

Del artículo citado se concluye que (i) el Estado es el obligado a ejecutar la consulta, (ii) los sujetos quienes deben ser consultados son aquellos pueblos interesados; es decir, pueblos indígenas o tribales a partir de lo regulado en el artículo 1 del Convenio 169, (iii) la consulta se debe ejecutar cada vez que alguna medida legislativa o administrativa pueda afectar a los mencionados pueblos directamente; y (iv) el objetivo de la consulta es lograr el consentimiento acerca de las medidas propuestas.

Posteriormente, el 13 de setiembre de 2007, mediante Resolución 61/295 de la Asamblea General, se aprobó la Declaración de las Naciones Unidas sobre los Derechos de los Pueblos Indígenas adoptada por el Consejo de Derechos Humanos el 29 de junio de 2006. El objetivo de la Declaración fue promover, en un marco de trabajo internacional, los derechos de los Pueblos Indígenas u Originarios tales como: igualdad y no discriminación, identidad e integridad cultural, acceso a tierras, territorios y recursos naturales, participación y consulta, como los derechos colectivos de los Pueblos Indígenas u Originarios, así como establecer el principio de autodeterminación de estos pueblos.

Tomando en cuenta lo anterior, para llevar a cabo la consulta, el Estado es quien deberá responder dos interrogantes:

1. Si está frente a un pueblo indígena $u$ originario; $y$

2. Si cuenta con una medida legislativa o administrativa que pueda afectarlos.

1 Artículo 56.- Los tratados deben ser aprobados por el Congreso antes de su ratificación por el Presidente de la República, siempre que versen sobre las siguientes materias:

1. Derechos Humanos.

2. Soberanía, dominio o integridad del Estado.

3. Defensa Nacional.

4. Obligaciones financieras del Estado.

También deben ser aprobados por el Congreso los tratados que crean, modifican o suprimen tributos; los que exigen modificación o derogación de alguna ley y los que requieren medidas legislativas para su ejecución.

2 Artículo 55.-Los tratados celebrados por el Estado y en vigor forman parte del derecho nacional. 
Las respuestas a estas dos interrogantes no se encuentran directamente establecidas en el Convenio 169 ya que este instrumento no establece una definición exacta respecto a quienes son los pueblos indígenas o tribales; y tampoco define lo que se debe entender por "medida legislativa" o "medida administrativa". Del mismo modo, no se regula el procedimiento que debía seguirse para hacer efectivo el proceso de consulta. Por lo tanto, la sola ratificación del Convenio 169, aun cuando forma parte de nuestra regulación, no permitió su aplicación inmediata pues sólo establece pautas generales a considerar.

En este escenario, no cabe duda que, frente a estas interrogantes, era obligación de cada uno de los Estados desarrollar el marco normativo necesario para que sea posible efectivizar el procedimiento de consulta y; con ello, el derecho en sí mismo. Lo anterior no significa que, frente a la falta de regulación interna, el Estado se encontraba exento de hacer valer el derecho. Por el contrario, partiendo del análisis efectuado por el Tribunal Constitucional ${ }^{3}$ y de la Corte Suprema ${ }^{4}$, la obligación de consultar existió a partir de la entrada en vigencia del Convenio 169.

Considerando lo anterior, a nivel nacional, entre la entrada en vigencia del Convenio 169 hasta la Ley del Derecho a la Consulta Previa a los Pueblos Indígenas u Originarios, el Estado (específicamente, el Ministerio de Energía y Minas) promulgó diversas normas que advertían un intento por incorporar en la legislación nacional las obligaciones asumidas al amparo del Convenio 169.

Específicamente, considerando el sector eléctrico, estas normas se vinculan con la regulación el procedimiento de participación ciudadana cuya finalidad es la de informar a la población en general sobre los alcances de un proyecto de inversión.

Así tenemos a las normas siguientes:

i. Reglamento de Consulta y Participación Ciudadana en el Procedimiento de aprobación de los Estudios Ambientales en el Sector Energía y Minas, aprobado por Resolución Ministerial No. 596-2002-EM-DM publicada el 21 de diciembre de 2002.

Este Reglamento definió el procedimiento de consulta previa en el numeral 3.1 del artículo 3 como aquel “[c]onstituido por talleres previos, que serán convocados por la DGAA [refiriéndose a la Dirección General de Asuntos Ambientales] en coordinación con la Autoridad Regional del lugar en donde se pretende desarrollar el proyecto minero o energético, que serán realizados dependiendo de la magnitud e importancia del

3 A manera de ejemplo se encuentra la Sentencia del Tribunal Constitucional: 22-2009PI/TC- 17 de junio de 2010. “[...] han transcurrido más de 15 años de su entrada en vigencia, tiempo suficiente para su regulación, lo que no ocurrió por exclusiva responsabilidad del Estado"; y, de otro lado porque "no es un argumento constitucionalmente válido excusar la aplicación de derechos fundamentales debido a una ausencia de regulación legal o infra legal". (énfasis agregado)

4 Sentencia A.P. No. 2232-2012-LIMA (23 de mayo de 2013) Fundamento 5.3 .- “Con la entrada en vigencia del Tratado [refiriéndose al Convenio 169], el Estado Peruano se encuentra obligado a cumplir el Convenio 169 de buena fe, conforma al "pacta sunt servanta"; para lo cual el Estado no puede invocar el derecho interno para justificar el incumplimiento del tratado; ello , de acuerdo a los artículos 26 y 27.1 de la Convención de Viena [...]; de lo que se extrae el carácter vinculante del Convenio 169, así como la exigibilidad y responsabilidad internacional para el Estado Peruano en el cumplimiento de las normas y derechos que el convenio reconoce". (énfasis agregado) 
proyecto" (énfasis agregado). Como el lector podrá advertir, la definición del alcance de la consulta previa no atiende a los aspectos del Convenio 169 debido a que, no distingue su ejecución respecto de pueblos indígenas $\mathrm{u}$ originarios, ni tampoco la medida administrativa en consulta, sino que se dispone que el proyecto en sí mismo será puesto a disposición para que las personas opinen sobre sus alcances dependiendo de su magnitud. Adicionalmente, no era el Estado, sino el privado, el llamado a ejecutar los mecanismos de participación ciudadana -distinto

Por lo tanto, no es posible concluir que esta norma efectivamente represente la puesta en práctica de los alcances del Convenio 169.

ii. Lineamientos para la Participación Ciudadana en las Actividades Eléctricas, aprobado por Resolución Ministerial No. 223-2010-MEM/DM publicada el 26 de mayo de 2010.

El artículo 1 de la norma bajo análisis indicó que su objetivo era

“[...] establecer los lineamientos necesarios para el desarrollo de los procedimientos de Consulta y mecanismos de Participación Ciudadana que son aplicables durante la tramitación de procedimientos relacionados al otorgamiento de derechos eléctricos, durante la elaboración y evaluación de los Estudios Ambientales; y durante el seguimiento y control de los aspectos ambientales de los Proyectos y Actividades Eléctricas".

Nuevamente esta norma tenía como objetivo regular los procedimientos de consulta, pero amplía su ámbito de aplicación al total desarrollo de un proyecto eléctrico, sin determinar con certeza la medida administrativa que requeriría de consulta. Efectivamente, nos permite concluir que existía una confusión entre el alcance de los procedimientos de participación ciudadana regulados para la participación del publico en general en el procedimiento de evaluación ambiental de un proyecto de inversión con la consulta previa que debía ser ejecutada únicamente respecto a la aprobación de medidas administrativas que afecten directamente a pueblos indígenas $\mathrm{u}$ originarios.

Téngase en cuenta que la evaluación de impacto ambiental, así como cualquier otro instrumento ambiental aplicable a un proyecto de inversión, incluyendo, pero no limitándose a un proyecto de inversión en el sector eléctrico, no permite la ejecución de ninguna actividad al no ser un título habilitante propiamente dicho.

iii. Reglamento del Procedimiento para la Aplicación del Derecho de Consulta a los pueblos indígenas para las Actividades Minero Energéticas, aprobado por Decreto Supremo No. 023-2011-EM publicado el 12 de mayo de 2011.

De acuerdo con el artículo 1 del Reglamento bajo análisis, su objetivo fue regular el procedimiento para la aplicación del derecho a la consulta de los pueblos indígenas para las actividades minero energéticas, de conformidad con los principios y reglas establecidos en los numerales 1 y 2 del articulo 6 y numeral 2 del artículo 15 del Convenio No. 169 de la OIT.

Considerando lo anterior, la finalidad de ejecutar la consulta era "[...] llegar a un acuerdo con los pueblos indígenas sobre las medidas del Sector Minero Energético [...], susceptibles de afectarlos directamente. Para tal efecto, el 
Estado deberá determinar si los intereses de los pueblos indígenas serían perjudicados directamente y en qué medida".

Adicionalmente, este Reglamento reguló los principios y procedimientos para realizar la consulta previa. Tanto es así que en su artículo 18 reguló que para el sector electricidad "[s]on medidas administrativas susceptibles de afectar directamente a los pueblos indígenas y, por tanto, es materia de consulta: el otorgamiento de concesiones temporales y definitivas de generación y trasmisión eléctrica; así como, el otorgamiento de autorización de centrales de generación termoeléctrica" (énfasis agregado).

En consecuencia, es recién con este Reglamento que se estableció un marco específico para la aplicación del derecho a la consulta previa de acuerdo con lo establecido en el Convenio 169. Ello, considerando que la Primera Disposición Transitoria reguló que "[l]os procedimientos administrativos iniciados antes de la vigencia de la presente norma se regirán por las normas de participación ciudadana vigentes en dicha oportunidad". (énfasis agregado)

Considerando lo anterior, antes de la entrada en vigencia del Reglamento del Procedimiento para la Aplicación del Derecho de Consulta a los Pueblos Indígenas para las Actividades Minero Energéticas, la regulación aprobada por el Ministerio de Energía y Minas había regulado la consulta previa a través de mecanismos de participación ciudadana sin establecer una clara distinción entre ambos procedimientos. Es a partir del mencionado Reglamento que estos dos tipos de procedimientos se diferencias totalmente, distinguiendo (i) las personas a quienes se dirigía y (ii) las medidas administrativas respecto de las cuales se aplicaba.

Es en este contexto que, el 7 de setiembre de 2011 que se publicó la Ley No. 29785, Ley del derecho a la consulta previa a los pueblos indígenas u originarios ("Ley"). Posteriormente, el 3 de abril del 2012 se publicó el Reglamento de la Ley, aprobado por Decreto Supremo No. 001-2012-MC (“DS001”). En el marco de esta regulación, se desarrolló el contenido, los principios y el procedimiento de aplicación del derecho a la consulta previa a los pueblos indígenas y originarios.

Así, con el artículo 1 de esta Ley, su objetivo fue "desarrollar el contenido, los principios y el procedimiento del derecho a la consulta previa a los pueblos indígenas $\mathbf{u}$ originarios respecto a las medidas legislativas que les afecten directamente" (énfasis agregado). Como se puede advertir, el artículo citado se asemeja en gran medida al alcance del artículo 6 del Convenio 169 antes analizado. En ese sentido, queda establecido que es con la entrada en vigencia de esta Ley, que efectivamente el Estado buscó regular las formas de ejecutar a cabalidad los alcances del Convenio 169.

El artículo 2 de la Ley reguló específicamente que el derecho a la consulta es ostentado por los pueblos indígenas $u$ originarios quienes deben ser consultados de forma previa sobre las medidas legislativas o administrativas que afecten directamente sus derechos colectivos, existencia física, identidad cultural, calidad de vida o desarrollo. Dicho derecho también incluye la obligación de consultar "[1]os planes, programas o proyectos de desarrollo nacional y regional que afecten directamente los derechos colectivos de los pueblos indígenas u originarios". Advertimos que la Ley establece una lista cerrada de temas que pueden significar una afectación directa a los pueblos indígenas. Por lo tanto, frente a una medida administrativa o legislativa que pueda afectar directamente a los pueblos indígenas $\mathrm{u}$ originarios deberá analizarse si tal medida representa una 
perturbación a sus derechos colectivos respecto de los temas específicamente descritos.

De acuerdo con el artículo 3 de la Ley, la finalidad de la consulta es alcanzar un acuerdo o consentimiento entre el Estado y los pueblos indígenas u originarios. Sin embargo, en caso de no llegar a un acuerdo, no se invalida el proceso de consulta previa. Ello, debido a que el artículo 15 de la citada Ley establece que la decisión final sobre la aprobación o no, de la medida consultada le corresponde a la entidad estatal competente. De esta manera, en este escenario, la entidad estatal debe adoptar las medidas que resulten necesarias para garantizar los derechos colectivos y los derechos a la vida, integridad y pleno desarrollo de los pueblos indígenas $\mathrm{u}$ originarios ${ }^{5}$. Por lo tanto, la ejecución de la consulta no es competencia del privado (como sí la ejecución de los procedimientos de participación ciudadana) sino del Estado quien debe determinar los casos en los que se cumplan con los elementos objetivos y subjetivos necesarios para que se ejecute una consulta previa.

A partir de lo anterior cabe concluir que, a partir de la ratificación del Convenio 169 se ha encontrado vigente el derecho a la consulta a favor de los pueblos indígenas u originarios. En este sentido, el Estado desarrollo una vasta regulación con el objetivo de hacer efectivo el derecho de la consulta previa. Sin embargo, es desde la promulgación de la Ley en el 2011 y, con mayor precisión, desde la entrada en vigencia del DS001 en el 2013, que efectivamente se ha venido implementando el derecho a la consulta con mayor precisión respecto a los alcances del Convenio 169.

\section{La identificación de una medida administrativa y la ejecución de la consulta previa en el sector eléctrico}

Atendiendo a los alcances de la Ley y el DS001, el Ministerio de Cultura - como autoridad competente para conducir a política de consulta previa, en palabras de Iván Lanegra6-, junto con doctrina nacional tal como La Rosa7 entre otros, establecen las siete etapas mínimas del proceso de consulta previa, dentro de las cuales se encuentra la identificación de la medida administrativa (o legislativa) cuya aprobación debe ser consultada ${ }^{8}$. Es sobre esta etapa que profundizaremos en la presente sección con la finalidad de determinar los alcances de esta identificación en el sector eléctrico.

\section{i. Los alcances de la medida correctiva a partir de la regulación en materia de consulta previa}

\footnotetext{
Artículo 15 de la Ley.

6 LANEGRA, Iván. "La Consulta Previa de la Construcción y Mantenimiento de la Infraestructura para los Pueblos Indígenas". En Forseti. Edición 2016, número 1.

7 LA ROSA, Javier. "El Derecho a la Consulta Previa y su implementación en el Perú según las Reglas Legislativas y el Tribunal Constitucional. En Derecho E Sociedad No. 39. Lima, 2012, pp. 196-203.

8 Atendiendo al Manual sobre Derecho A la Consulta Previa elaborado por el Ministerio de Cultura. Las siete etapas son las siguientes: Identificación de la medida administrativa (o legislativa) que debe ser consultada, identificación de los pueblos indígenas $u$ originarios, publicidad de la medida administrativa (o legislativa), información sobre la medida administrativa (o legislativa), evaluación interna en las instituciones y organizaciones de los pueblos indígenas $\mathrm{u}$ originarios sobre la medida administrativa (o legislativa) que les afecte directamente, proceso de diálogo entre los representantes del Estado y los representantes de los
} pueblos indígenas u originarios; y la toma de decisión. 
En base a un análisis en conjunto de los artículos 2 y 9 de la Ley, cabe concluir que la sola identificación de una medida administrativa que se vaya a aprobar respecto a la ejecución de actividades y/o un proyecto que se ubique sobre el área en la que se encuentra un pueblo indígena $u$ originario no es suficiente para tener que activar el proceso de consulta previa. Es necesario, además, confirmar la existencia de derechos colectivo indígenas comprometidos y la determinación de su afectación. Caso contrario, no sería necesario ejecutar el proceso de consulta previa.

Efectivamente, de acuerdo con el artículo 9 de la Ley, la obligación de implementar el derecho de consulta recae en aquellas entidades del Estado que van a emitir medidas legislativas o administrativas relacionadas de forma directa con los derechos de los pueblos indígenas u originarios. Por ejemplo, en el sector eléctrico, el Ministerio de Energía y Minas es el encargado en emitir la medida administrativa que efectivamente podría afectar a los pueblos indígenas $u$ originarios, de manera positiva o negativa.

En ese sentido, esta etapa consiste en la identificación de la medida a consultar por parte de las entidades públicas con la finalidad de determinar si tal medida podría afectar sus derechos colectivos. Por ello, resulta necesario determinar lo que debe entenderse por medida administrativa y sus excepciones.

Sobre el concepto de "medida administrativa", el artículo 3 del DS001 la define como la "[...] norma reglamentaria de alcance general, así como el acto administrativo que faculte el inicio de la actividad o proyecto, o el que autorice a la Administración la suscripción de contratos con el mismo fin, en tanto puedan afectar directamente los derechos colectivos de los pueblos indígenas". (énfasis agregado)

En esta línea, la Décima Quinta Disposición Complementaria, Transitoria y Final del DS001 reguló que "[1]a construcción y mantenimiento de infraestructura en materia de salud, educación, así como la necesaria para la provisión de servicios públicos que, en coordinación con los pueblos indígenas, esté orientada a beneficiarlos, no requerirán ser sometidos al procedimiento de consulta previsto en el Reglamento" (énfasis agregado). En consecuencia, mediante esta Disposición se reguló que la provisión de servicios públicos será una medida que no requerirá ser sometida al procedimiento regular de consulta previa. Por el contrario, al determinar que la prestación del servicio supone un beneficio para los pueblos indígenas (a partir de la coordinación con ellos), se podrá permitir la ejecución del proyecto sin que se someta a este procedimiento.

En palabras de Lanegra, esta excepción a la aplicación del procedimiento "regular" de consulta previa supone la aplicación del principio de flexibilidad de la Ley. En su opinión, " [n]o es una excepción del deber de consultar - cuestión que no podría ser creada mediante Decreto Supremo y que tendría que tener base legal- sino de una excepción al uso del procedimiento establecido en el propio reglamento [refiriéndose al DS001]"9.

En todo caso, con la finalidad de profundizar el alcance de la excepción regulada en el Disposición antes descrita, el 27 de mayo de 2016, el Viceministerio de Interculturalidad del Ministerio de Cultura emitió la Resolución Viceministerial

9 LANEGRA, Iván. “La Consulta Previa de la Construcción y Mantenimiento de la Infraestructura para los Pueblos Indígenas". En Forseti. Edición 2016, número 1. 
No. 013-2016-VMI-MC, que aprobó la Directiva 001-2016-VMI/MC "Procedimientos para la Aplicación de lo Dispuesto en la Décima Quinta Disposición Complementaria, Transitoria y Final del Reglamento de la Ley 29785, Ley del Derecho a la Consulta Previa a los Pueblos Indígenas u Originarios, reconocido en el Convenio 169 de la Organización Internacional del Trabajo".

Esta Directiva estableció los aspectos que comprenden la aplicación de la Décima Quinta Disposición Complementaria, Transitoria y Final del DS001 antes mencionada, diferenciando los alcances de la provisión de servicios públicos en los términos siguientes:

“[...] la construcción y mantenimiento de infraestructura que se realiza de manera directa, a través de una entidad administrativa, o indirecta, a través de operaciones privados, para la satisfacción de la demanda colectiva en las poblaciones.

La sola construcción y mantenimiento de infraestructura destinada a la provisión de Servicios Públicos genera beneficios en la población, en la medida que busca satisfacer y garantizar el ejercicio de derechos fundamentales de la población. Dicho beneficio no es efectivo si no se garantiza la continuidad y calidad del servicio, situación que se concreta con las actividades de mantenimiento". (énfasis agregado)

Es decir, a partir de lo desarrollado por el Viceministerio de Interculturalidad del Ministerio de Cultura, un servicio público que no requeriría la ejecución de un procedimiento de consulta previa pero la participación de los pueblos indígenas beneficiados a través de un Plan Informativo, son aquellas construcciones que satisfacen la demanda colectiva de la población, les generan beneficios; y buscan satisfacer y garantizar el ejecución de derechos fundamentales.

Advertimos que, autores con Lanegra han afirmado que la Directiva bajo consulta ignora los alcances del DS001 en la medida que no dispone la aplicación de un procedimiento simplificado de consulta sino que exceptúa la ejecución de éste procedimiento e incluso deja de regular una fase de identificación del beneficio que generaría la actividad (en este caso la implementación del servicio público). De esta manera, la Directiva vendría a ser contraria a las disposiciones de rango constitucional, pudiendo interponerse acciones legales en contra de las medidas aprobadas sin la ejecución de un procedimiento de consulta previa "simplificado" 10.

En todo caso, concluimos que la opinión brindada es una postura que bien puede ser cuestionable pues allí donde dice "excepción a la consulta" no puede interpretarse "excepción a un proceso específico por otro". Lo anterior no supone rechazar la necesidad de hacer efectivo los derechos de los pueblos indígenas $\mathrm{u}$ originarios, sino ejecutar los procesos de consulta respecto de situaciones que efectivamente lo ameriten. Considerando ello, se entiende que, en la práctica, la Disposición antes descrita es sustento suficiente para que no se ejecute un procedimiento de consulta previa y en su lugar se apruebe un Plan Informativo, considerando los alcances de la mencionada Directiva.

10 Ídem. 


\section{ii. Análisis de las medidas administrativas del sector eléctrico11}

Tomando en cuenta lo anterior, en el sector eléctrico, será necesario determinar cuál es la medida administrativa cuya aprobación puede suponer la afectación de pueblos indígenas $u$ originarios (de haberse identificado).

$\mathrm{Al}$ respecto, la Ley de Concesiones Eléctricas, aprobada por Decreto Ley No. 25844 -aprobada con fecha anterior a la Ley y el DS001, regula como actividades eléctricas, la generación, transmisión, distribución y comercialización de la energía eléctrica ${ }^{12}$.

En este escenario, el artículo 2 de la mencionada norma establece que tanto (i) el suministro regular de energía eléctrica para uso colectivo o destinado al uso colectivo, hasta los límites de potencia fijados por el Reglamento; como, (ii) la transmisión y distribución de electricidad, son servicios públicos de electricidad. Por lo tanto, atendiendo a los alcances de la Décima Quinta Disposición Complementaria, Transitoria y Final del DS001 y la Directiva 001-2016-VMI/MC antes analizadas, tales actividades no requerirían la ejecución de un procedimiento de consulta previa pero de la presentación de un Plan Informativo, al ser consideradas como "servicios públicos" 13 .

Considerando ello, resulta necesario analizar si las actividades de generación eléctrica y comercialización - las cuales no han sido reguladas como servicios públicos - suponen la aprobación de una medida administrativa que amerite la puesta en marcha de un proceso de consulta previa.

Sobre la generación eléctrica, esta actividad “[...] es la primera de [...] la cadena productiva de energía eléctrica, la cual consiste en transformar alguna clase de energía (térmica, mecánica, luminosa, entre otras) en energía eléctrica"14. Existen diversos tipos que pueden ser clasificados en función a la fuente de energía primaria que utilizan tal como la hidráulica, el petróleo, gas natural, entre otras. En este contexto, el título habilitante que permite la ejecución de actividades de generación es la concesión definitiva y la autorización, dependiendo del tipo de generación.

Efectivamente, el artículo 3 de la Ley de Concesiones Eléctricas advierte que

"[s]e requiere concesión definitiva para el desarrollo de cada una de las siguientes actividades: a) La generación de energía eléctrica que utilice recursos hidráulicos, con potencia instalada mayor de $500 \mathrm{KW}$; [...] d) La generación de energía eléctrica con recursos energéticos renovables conforme a la Ley de la materia, con potencia instalada mayor a 500 KW" (énfasis agregado).

11 Advertimos que no se analizan los permisos transversales tales como: instrumentos ambientales, permisos vinculados con el manejo de patrimonio arqueológico, derechos en materia de recursos hídricos, entre otros.

12 Artículo 1 de la Ley de Concesiones Eléctricas.

13 De acuerdo con el fundamento 40 de la Sentencia con Expediente No. 00034-2004-AI/TC, los elementos que en conjunto permiten clasificar a un servicio como público son los siguientes: (i) su naturaleza es esencial para la comunidad, (ii) la necesaria continuidad de su prestación en el tiempo, (iii) su naturaleza regular, es decir, que debe mantener un estándar mínimo de calidad, y (iv) la necesidad de que su acceso se dé en condiciones de igualdad.

14 LIRA, Alfredo, Fiorella MOLINELLI y Max Arturo CARBAJAL. Fundamentos Técnicos y Económicos del Sector Eléctrico Peruano. Lima: Organismo Supervisor de la Inversión en Energía y Minería. 2011, p.21. 
Adicionalmente, el artículo 4 de la citada Ley regula que "[s]e requiere autorización para desarrollar las actividades de generación termoeléctrica, cuando la potencia instalada sea superior a 500 KW" (énfasis agregado)

Por otro lado, sobre la actividad de comercialización, esta no requiere del otorgamiento de un título habilitante (i.e., medida administrativa) previa a su ejecución. Por lo tanto, no se genera el escenario en el que sea necesaria la ejecución de un procedimiento de consulta previa.

En este contexto, tomando en cuenta los títulos habilitantes mencionados, el 20 de julio de 2012, el Ministerio de Energía y Minas aprobó la Resolución Ministerial No. 350-2012-MEM/DM aprobó los procedimientos administrativos en los que corresponde realizar el proceso de consulta previa, así como la oportunidad en la que debe realizarse y a la Dirección General del Ministerio de Energía y Minas encargada de su ejecución, modificados luego mediante la Resolución Ministerial No. 209-2015-MEM/DM la cual atiende a la excepción incluida en la Décima Quinta Disposición Complementaria, Transitoria y Final del DS001.

En consecuencia, a la fecha, para el sector electricidad se requiere ejecutar un procedimiento de consulta previa de acuerdo con el detalle siguiente ${ }^{15}$ :

\begin{tabular}{|c|c|c|}
\hline $\begin{array}{c}\text { Procedimiento } \\
\text { Administrativo }\end{array}$ & $\begin{array}{c}\text { Oportunidad del proceso } \\
\text { de consulta }\end{array}$ & Dirección a cargo \\
\hline $\begin{array}{c}\text { Otorgamiento de } \\
\text { concesión definitiva de } \\
\text { generación, transmisión y } \\
\text { distribución }\end{array}$ & $\begin{array}{c}\text { Antes de otorgar la } \\
\text { concesión }\end{array}$ & $\begin{array}{c}\text { Dirección General de } \\
\text { Asuntos Ambientales } \\
\text { Energéticos }\end{array}$ \\
\hline $\begin{array}{c}\text { Otorgamiento de } \\
\text { Concesión Rural16 }\end{array}$ & $\begin{array}{c}\text { Antes de otorgar la } \\
\text { concesión }\end{array}$ & $\begin{array}{c}\text { Dirección General de } \\
\text { Asuntos Ambientales } \\
\text { Energéticos }\end{array}$ \\
\hline $\begin{array}{c}\text { Modificación de concesión } \\
\text { definitifiva de generación } \\
\text { y transmisión (sólo si } \\
\text { implica ocupación de } \\
\text { nuevas áreas) }\end{array}$ & Antes de otorgar la \\
concesión & $\begin{array}{c}\text { Dirección General de } \\
\text { Asuntos Ambientales } \\
\text { Energéticos }\end{array}$ \\
\hline $\begin{array}{c}\text { Outorización para } \\
\text { exploración de recursos } \\
\text { geotérmicos }\end{array}$ & $\begin{array}{c}\text { Antes de la autorización } \\
\text { Autorización de } \\
\text { generación termoeléctrica }\end{array}$ & $\begin{array}{c}\text { Antes de la autorización } \\
\text { Asuntos Ambientales } \\
\text { Energéticos }\end{array}$ \\
\hline & $\begin{array}{c}\text { Asuntos Ambientales } \\
\text { Energéticos }\end{array}$ \\
\hline
\end{tabular}

15 Artículo 1 de la Resolución Ministerial No. 209-2015-MEM/DM.

16 El análisis de los títulos habilitantes y procedimientos vinculados con la actividad de electrificación rural se encuentra fuera del alcance del presente artículo. 


\begin{tabular}{|c|c|c|}
\hline $\begin{array}{c}\text { Otorgamiento de } \\
\text { Concesión para } \\
\text { explotación de recursos } \\
\text { geotérmicos }\end{array}$ & $\begin{array}{c}\text { Antes de otorgar la } \\
\text { concesión }\end{array}$ & $\begin{array}{c}\text { Dirección General de } \\
\text { Asuntos Ambientales } \\
\text { Energéticos }\end{array}$ \\
\hline
\end{tabular}

A partir de lo anterior queda establecido que, el Ministerio de Energía y Minas, como autoridad competente sobre la regulación del sector eléctrico ha determinado que para la ejecución de la actividad de generación se requerirá consulta previa antes del otorgamiento de la concesión definitiva o autorización que la permita.

Considerando ello, de acuerdo con la información pública brindada por el Ministerio de Energía y Minas, cuatro proyectos vinculados con el sector electricidad habrían sido participes del proceso de consulta previa: (i) Central Hidroeléctrica La Herradura - El Gallo, (ii) Central Hidroeléctrica Pachachaca 3, (iii) Central Hidroeléctrica Río Araza S.A.C.; y (iv) Centrales Hidroeléctrica Anto Ruiz III y IV17. Como puede advertirse de la propia denominación de los Proyectos, todos versan sobre la implementación de una central.

Aun cuando la regulación ha determinado la medida administrativa que debe ser materia de consulta frente a un proyecto del sector de electricidad, es importante plantear el cuestionamiento de si efectivamente el otorgamiento de la concesión definitiva y/o la autorización representa el "acto administrativo que faculta el inicio de la actividad o proyecto".

En la práctica podemos advertir que, la concesión no faculta el inicio de la actividad, sino la operación de la infraestructura eléctrica ya construida. Sin lugar a duda, la construcción de tal infraestructura genera una afectación que no es evaluada por la autoridad competente a efectos de determinar si es en este momento que la consulta previa puede ejecutarse (de aplicar). Resultará necesario entonces evaluar el proceso de implementación de un proyecto eléctrico con la finalidad de determinar si efectivamente el otorgamiento del título habilitante en consulta constituye la medida administrativa que debería gatillar el procedimiento de consulta previa.

\section{iii. Proyectos eléctricos en los que se ha identificado la necesidad de iniciar un proceso de consulta previa}

Como se identificó en la sección ii., anterior, de acuerdo con la información pública del Ministerio de Energía y Minas, cuatro Proyectos habrían requerido el inicio de un procedimiento de consulta previa: a) Central Hidroeléctrica La Herradura - El Gallo, b) Central Hidroeléctrica Pachachaca 3, c) Central Hidroeléctrica Río Araza S.A.C.; y d) Centrales Hidroeléctrica Anto Ruiz III y IV18 (los "Proyectos").

Como punto de partida importa identificar que los Proyectos versan sobre la operación de Centrales Hidroeléctricas. Por lo tanto, queda establecido que, en atención a la regulación aplicable, no se ha cuestionado la necesidad de ejecutar el proceso de consulta respecto de proyectos que supongan la ejecución de actividades de transmisión ni suministro de energía.

17 Ingreso al portal web del Ministerio de Energía y Minas: 24 de octubre de 2019.

18 Ingreso al portal web del Ministerio de Energía y Minas: 24 de octubre de 2019. 
$\mathrm{Al}$ respecto, advertimos que este es un tema controvertido se han establecido diversos cuestionamientos respecto de la exclusión regulada en la Décima Quinta Disposición Complementaria, Transitoria y Final del DS001. Así, el Instituto de Defensa Legal (IDL) ha cuestionado, por ejemplo que, esta exclusión nacida de un reglamento viola el principio de jerarquía reconocido en los artículos 51 y 138 de la Constitución Política del Perú debido a que "modifica" los alcances del Convenio 169 (norma con rango constitucional) ${ }^{19}$.

Sobre esta base, a continuación analizaremos los casos que han ameritado la ejecución de procedimientos de consulta previa en el sector eléctrico diferenciando brevemente sus etapas y acuerdos adoptados con la finalidad de otorgar al lector una idea general sobre el análisis ejecutado por la autoridad encargada de ejecutar el proceso de consulta previa a proyectos de inversión en el sector eléctrico.

\section{a) Central Hidroeléctrica La Herradura - El Gallo}

De acuerdo con el Plan de Consulta del proyecto materia de análisis, se sometió a consulta la aprobación de la concesión definitiva de generación de energía del proyecto "Central Hidroeléctrica La Herradura - El Gallo" debido a que "[e]l Ministerio de Energía y Minas, definió el ámbito de la medida, considerando el área de influencia directa del proyecto "Central Hidroeléctrica La Herradura-El Gallo", que involucra a las comunidades campesinas de San Miguel de Huacá Puna, San Juan de Yarucaya y Santo Tomás de Cochamarca".

Al respecto advertimos que la identificación de los pueblos originarios a ser consultados partió de un trabajo de campo y gabinete realizado por el Ministerio de Cultura.

Sobre la medida a consultar, el Ministerio de Energía y Minas únicamente concluyó que, a partir de la regulación en materia de consulta previa (analizadas líneas arriba) el otorgamiento de una concesión definitiva debe per se es materia de consulta. Cabe resaltar que la medida que se presenta para consulta es solo el proyecto de Resolución de aprobación de la concesión y no el expediente técnico. De hecho, el que se presente el documento ya finalizado en realidad resulta incongruente respecto con la finalidad del procedimiento de consulta previa.

Efectivamente, existe poco margen de decisión respecto a cómo la aprobación de esta medida podría variar si es que todos los aspectos técnicos y legales ya han sido atendidos por el Ministerio. En todo caso, bien podría ser recomendable ejecutar este procedimiento en paralelo al análisis de la información por la autoridad competente (similar a la aplicación de los procedimientos de participación ciudadana al momento de evaluar in instrumento de gestión ambiental), con la finalidad de que efectivamente se incorporen compromisos mutuos, de ser necesario.

Finalmente, el presente procedimiento de consulta previa concluyó con el Acta de Consulta Previa del Proyecto materia de análisis. Resulta necesario advertir que

19 Este argumento ha sido incluido en el marco del proceso de amparo presentado por la Organización Regional de los Pueblos Indígenas del Oriente (ORPIO) Y LA Coordinadora Regional de los Pueblos Indígenas de la Región San Lorenzo, contra el Ministerio de Energía y Minas y Proinversión por omisión de consulta del Proyecto Línea de Transmisión Eléctrica Moyobamba-Iquitos. [A la fecha, esta demanda fue desestimada] https://idl.org.pe/por-quedebe-confirmarse-la-sentencia-que-ordena-la-consulta-del-proyecto-linea-de-transmisionelectrica-moyobamba-iquitos/ 
en la etapa de dialogo entre la entidad promotora y los miembros de las comunidades no sólo se trataron los alcances de la medida administrativa sujeta a evaluación. Por el contrario, también se dialogaron diversas propuestas no relacionadas a la concesión definitiva tales como: represamiento de lagunas, la necesidad de contar con un servicio de agua potable, la puesta en marcha de proyectos que mejoren la salud de las personas.

A partir de lo anterior, es posible concluir que el mecanismo de consulta previa, al buscar entablar un diálogo para determinar la posibilidad de aprobar o no una medida administrativa, ha abierto la puerta a la escucha del estado por los problemas sociales que aquejan a este sector de la población.

Tomando en cuenta que la población aceptó la aprobación de la medida administrativa, se dio por concluido el procedimiento de consulta.

\section{b) Central Hidroeléctrica Pachachaca 3}

Atendiendo al Plan de Consulta, el Ministerio de Energía y Minas consideró que el área de influencia directa del proyecto bajo análisis involucraba comunidades campesinas de Llactabamba, Túpac Amaru II y Juan Velasco Alvarado. Estas comunidades fueron identificadas como pueblos indígenas y originarios por el Ministerio de Cultura.

Advertimos que, de acuerdo con el Oficio No. 280-2018-MEM/DGAAE del 30 de abril de 2018, el Ministerio de Energía y Minas comunicó a las tres comunidades campesinas involucradas en el proceso de consulta previa que el administrado había decidido desistirse del procedimiento administrativo de Concesión Definitiva. En consecuencia, teniendo en cuenta que el proceso de consulta previa se realiza en relación a la solicitud de concesión definitiva, no cabía continuar con la consulta.

\section{c) Central Hidroeléctrica Río Araza S.A.C.}

Nuevamente, atendiendo al Plan de Consulta vinculado con este proyecto, la identificación de los pueblos indígenas u originarios ubicados en el ámbito de la medida (i.e., el otorgamiento de la concesión definitiva) fue ejecutada a través de la revisión de información de gabinete y también trabajo de campo con la finalidad de determinar criterios objetivos tales como: la continuidad histórica, conexión territorial, instituciones distintivas; y el criterio subjetivo de auto-identificación. El resultado de esta etapa permitió identificar la presencia de pueblos indígenas $u$ originarios en las comunidades campesinas de Marcapata Cccollana, Socapata y Unión Araza.

Advertimos que la medida administrativa se identificó a partir del criterio ya definido en la regulación analizada líneas arriba.

Como consecuencia de las reuniones preparatorias, publicidad de la medida materia de consulta y evaluación interna por los miembros de las comunidades involucradas, la etapa de dialogo dio como resultado la determinación de compromisos no relacionados con la medida administrativa pero vinculados con temas de educación, salud, actividades agropecuarias, servicios básicos y la dación de infraestructura. 
En ese sentido, nuevamente cabe advertir que aun cuando el proceso de consulta previa versa sobre la aprobación de una determinada medida administrativa (en este caso una que se vincula con el otorgamiento de una concesión definitiva para la generación de energía), los temas que se dialogan con mayor énfasis son medidas que no se vinculan con la medida administrativa pero el otorgamiento de servicios públicos básicos.

\section{d) Centrales Hidroeléctrica Anto Ruiz III y IV}

Las comunidades campesinas involucradas en el proceso de consulta fueron la de Carabaya y Kana. De acuerdo con la información pública del Ministerio de Energía y Minas, el desarrollo del presente proceso de consulta tuvo diversas reuniones preparatorias que dieron como resultado la propuesta de un Plan de Consulta.

Mediante el mencionado Plan se identificó la medida a consultar, así como a los pueblos indígenas involucrados.

Sobre la medida a consultar, al igual que en los casos anteriores, se identificó la regulación analizada líneas arriba lo cual permitió identificar que la concesión definitiva para desarrollar la actividad de generación de energía eléctrica del proyecto materia de análisis, ameritaba el inicio de un proceso de consulta previa.

Adicionalmente, sobre la identificación de los pueblos originarios a ser consultados, se determinó que el ámbito de aplicación de la medida administrativa afectaría a terrenos de las comunidades campesinas de Carabaya e Ituata. Estas comunidades cumplían con los requisitos necesarios para ser considerados como pueblos indígenas $\mathrm{u}$ originarios a partir de las conclusiones arribadas por el Ministerio de Cultura. Por esta razón se determinó que, la consulta procedía.

Advertimos que, de la revisión del Plan, no se analizó la afectación que esta medida podría generar en los pueblos mencionados. Únicamente se planteó que la concesión definitiva ameritaba la ejecución de un proceso de consulta y, tras la identificación de los pueblos, tal proceso se inició.

Así como en los casos anteriores, se determinó una mayor cantidad de compromisos no vinculados con el proyecto bajo análisis relacionados con el desarrollo de servicios públicos. En ese sentido, es un común denominador que los pueblos consultados utilicen esta oportunidad para tener un acercamiento con el Estado y trasladen sus preocupaciones, las cuales no se encuentran directamente vinculadas con la medida administrativa en consulta.

\section{Conclusiones}

El presente artículo ha tenido como finalidad analizar los alcances del derecho a la consulta y su aplicación en el sector eléctrico.

De esta manera, se ha analizado la regulación vinculada con la aplicación de este derecho en el mencionado sector. A partir de ello ha sido posible advertir que, inicialmente, hacer efectiva la aplicación del derecho a la consulta previa se ejecutó a través de procesos de participación ciudadana. Fue con la aprobación de la Ley y el DS001 que se independizó y profundizó la aplicación de este derecho. Lo anterior no significa que aquellas medidas administrativas aprobadas durante el periodo entre la entrada en vigencia del Convenio 169 y la Ley, sino que se entendió como efectivo a través de las normas de participación ciudadana. 
Adicionalmente, un aspecto trascendental a analizar ha sido determinar las medidas administrativas que requieren de consulta. A la fecha, el Ministerio de Energía y Minas ha determinado claramente que esta medida es la concesión definitiva. Excluyendo, actividades eléctricas que son calificadas como "servicios públicos". Lo anterior, sin embargo, no debe dejar de lado la posibilidad de cuestionar si efectivamente la concesión definitiva es una medida administrativa cuyo alcance deba efectivamente ameritar ser consultada, en lugar de, por ejemplo, otros permisos que en la practica efectivamente permitan el inicio del proyecto eléctrico.

Finalmente, advertimos que aun cuando el cuestionamiento anterior sea necesario, lo cierto es que, a la fecha, los procesos de consulta previa en el sector eléctrico siguen vinculados con la aprobación de concesiones definitiva de generación eléctrica. 